\title{
Crystal Structure and Interaction Dependence of the Crystal-Melt Interfacial Free Energy
}

\author{
Ruslan L. Davidchack \\ Department of Mathematics, University of Leicester, Leicester LE1 7RH, United Kingdom
}

Brian B. Laird

Department of Chemistry, University of Kansas, Lawrence, Kansas 66045, USA

(Received 23 November 2004; published 1 March 2005)

\begin{abstract}
We examine via molecular simulation the dependence of the crystal-melt interfacial free energy $\gamma$ on molecular interaction and crystal structure (fcc vs bcc) for systems interacting with inverse-power repulsive potentials, $u(r)=\epsilon(\sigma / r)^{n}, 6 \leq n \leq 100$. Both the magnitude and anisotropy of $\gamma$ are found to increase as the range of the potential increases. Also we find that $\gamma_{\mathrm{bcc}}<\gamma_{\mathrm{fcc}}$, consistent with recent observations that some fcc forming fluids nucleate via formation of metastable bcc nuclei. The anisotropy in $\gamma$ is also seen to be smaller in the bcc systems. By extrapolation, we also obtain an improved estimate of $\gamma$ for hard spheres.
\end{abstract}

DOI: 10.1103/PhysRevLett.94.086102

PACS numbers: 68.08.De, 05.70.Np, 81.10.Aj

The crystal-melt interfacial free energy $\gamma$ is the reversible work needed to form a unit area of interface between a crystal and its melt [1]. Both the magnitude and anisotropy of $\gamma$ are of significant importance in a number of disciplines, as they are the primary controlling parameters governing the kinetics and morphology of crystal growth [2]. For example, the nucleation rates of crystals $[3,4]$ and colloids [5] exhibit a strong dependence on $\gamma$, and its anisotropy has a profound effect on the nature of dendritic growth $[6,7]$. Unfortunately, direct experimental measurements for $\gamma$ exist for only a few materials [8], and, with the exception of a small number of studies $[9,10]$, the values determined are not of sufficient precision to resolve the anisotropy. The paucity of experimental data, together with a desire to understand the thermodynamics of crystal-melt interfaces from a molecular perspective, has motivated recent interest in the development of computational methods for the calculation of $\gamma[11,12]$. These efforts have focused both on the determination of $\gamma$ for realistic models of materials (especially metals [12-14] and metal alloys [15]) and for simple models, such as hard spheres [11] and Lennard-Jones particles [16-18].

The current understanding of the dependence of $\gamma$ on material properties is largely empirical. In 1950, Turnbull [19] reported values of $\gamma$ for a variety of materials, obtained indirectly from nucleation rate experiments. Turnbull observed a strong correlation between the enthalpy of fusion per particle $\Delta H_{\text {fus }}$ and $\bar{\gamma} \equiv \gamma \rho_{c}^{-2 / 3}$, where $\rho_{c}$ is the crystal number density and $\bar{\gamma}$ is a measure of the free energy per interfacial particle. This correlation (known as "Turnbull's rule") can be expressed mathematically as

$$
\bar{\gamma}=C_{T} \Delta H_{\text {fus }},
$$

where the Turnbull coefficient, $C_{T}$, is found to be approximately 0.45 for metals and 0.32 for many nonmetals. Several theories [20-22] have been advanced to explain the molecular origin of Eq. (1), but the results are quite sensitive to the nature of the assumed interfacial structure.
These theories have in common an assumption that the crystal-melt interfacial free energy is primarily entropic in origin. This approach is supported by the recent observation that $\gamma$ for fcc metals is well approximated using a hard-sphere model [23]. The hard-sphere model is, however, unable to describe the variability in $C_{T}$ and in the anisotropy across systems.

The dependence of $\gamma$ on crystal structure is crucial to understanding the role of metastable structures in nucleation pathways. In 1897, Ostwald [24] formulated his "step rule," which states that nucleation from the melt occurs to the phase with the lowest activation barrier, which is not necessarily the thermodynamically most stable bulk phase. In the case of the nucleation of face-centered-cubic (fcc) crystals, there is evidence that crystallization often proceeds first through the formation of body-centered-cubic (bcc) nuclei, which transform to fcc crystallites later in the growth process. This phenomenon has been observed in experiments on metal alloys [25], in computer simulations of Lennard-Jones particles [26] and weakly charged colloids [27], and in classical density-functional theory studies of nucleation in Lennard-Jones [28]. These results could be explained if $\gamma$ for bcc crystals were significantly lower than that for fcc crystals in these systems as that would lead to substantially lower activation barriers. Using a simple model of interfacial structure, Spaepen and Meyer [22] predicted that $\gamma$ for bcc-melt interfaces should be about $20 \%$ lower than that for fcc-melt interfaces, based on packing considerations. In a recent paper, Sun et al. [14] determined $\gamma$ for bcc- and fcc-melt interfaces for several models for iron, obtaining values of $\gamma$ and $C_{T}$ that were about 30\%-35\% smaller for bcc-melt interfaces than for the corresponding fcc interfaces.

In this work we examine, via molecular simulation, the interfacial free energies for a large number of systems interacting with inverse-power potentials of varying range, crystal orientation, and crystal structure (fcc or bcc). By focusing on a general class of model interaction potentials 
instead of on a single, specific system, we aim to develop a generic molecular-level understanding of the interfacial thermodynamics of simple materials.

The inverse-power series of purely repulsive potentials is defined by the pair interaction

$$
u(r)=\epsilon\left(\frac{\sigma}{r}\right)^{n},
$$

where $\epsilon$ and $\sigma$ set the energy and length scale, respectively. The range of the interaction decreases with increasing $n$, approaching the hard-sphere limit as $n \rightarrow \infty$. This potential series has a number of notable properties that make it useful for the current study. First, because there is only one parameter in the potential, $\epsilon \sigma^{n}$, the density and temperature scales are not independent and all excess thermodynamic quantities are functions only of the quantity [29]

$$
\Gamma_{n} \equiv \rho \sigma^{3}(k T / \epsilon)^{-3 / n}=\rho^{*} T^{*-3 / n} .
$$

In particular, the phase diagram for these systems is one dimensional, with coexistence fully specified by giving the coexistence values of $\Gamma_{n}$ in the crystal $\left(\Gamma_{n, c}\right)$ and fluid $\left(\Gamma_{n, f}\right)$ phases. As a result, both the reduced pressure $P^{*}$ and reduced interfacial free energy $\gamma^{*}$ along the coexistence boundary exhibit power-law scaling with the melting temperature:

$$
\begin{aligned}
& P^{*} \equiv P \sigma^{3} / \epsilon=P_{1} T^{* 1+3 / n}, \\
& \gamma^{*} \equiv \gamma \sigma^{2} / \epsilon=\gamma_{1} T^{* 1+2 / n},
\end{aligned}
$$

where the subscript " 1 " denotes the quantity at $T^{*}=1$. Second, the entropy of fusion, $\Delta S_{\text {fus }}$, for these systems is constant along the coexistence curve; therefore, the corresponding $\Delta H_{\text {fus }}$ is directly proportional to $T_{m}$.

The values of $\Gamma_{n, c}, \Gamma_{n, f}$, and $P_{1}$ at coexistence have been previously obtained via simulation for $6 \leq n \leq \infty$ (see Ref. [30], and references therein). For large $n$, the fluid freezes to an fcc crystal structure, but below about $n=7$, the bcc structure becomes the thermodynamically stable phase on freezing. Because the free energy difference between the fcc and bcc phases is small, metastable bcc (or fcc) crystal-melt interfaces are quite stable, even for long simulation runs. Thus, this potential series is a good candidate for comparing fcc and bcc values of $\gamma$ for fixed interaction potential.

A scaling expression for Turnbull's $\bar{\gamma}=\gamma \rho_{c}^{-2 / 3}$ can be obtained by combining Eqs. (3) and (5) to give

$$
\begin{aligned}
\bar{\gamma} & =\gamma^{*} \rho^{*-2 / 3}=\gamma_{1} T_{m}^{* 1+2 / n}\left(\Gamma_{n, c} T_{m}^{* 3 / n}\right)^{-2 / 3} \\
& =\left(\gamma_{1} \Gamma_{n, c}^{-2 / 3}\right) T_{m}^{*}=\bar{\gamma}_{1} T_{m}^{*} .
\end{aligned}
$$

Thus, $\bar{\gamma}$ scales with the melting temperature for all inversepower potentials, not just hard spheres. The scaling with $T_{m}$ occurs even though $\gamma$ for these systems is not purely entropic in origin. Because $\Delta H_{\text {fus }}$ scales with $T_{m}$ for these systems $\left[\Delta H_{\text {fus }}=\left(\Delta H_{\text {fus }, 1}\right) T_{m}\right]$, we have the interesting result that Turnbull's rule is exact for inverse-power po- tentials. Equation (6) has been shown to well approximate $\gamma$ for a variety of close-packed metals [23] (with a proportionality constant of about 0.5 , based on Turnbull's nucleation data [19]), and can be viewed as an alternative to Turnbull's rule.

At present there are two methods for the calculation of $\gamma$ via simulation. In the fluctuation method [12] the interfacial stiffness is obtained from the spectrum of interfacial fluctuations [12]. By determining the stiffness for a variety of interfacial orientations, accurate values of $\gamma$ can be obtained. In the cleaving method $[11,16]$, which is employed here, $\gamma$ is obtained using thermodynamic integration-directly calculating the reversible work per unit area required to continuously transform separate bulk crystal and melt systems into a single system containing an interface. The cleaving procedure consists of four basic steps: (i) cleave the crystal system with a suitably chosen "cleaving" wall potential, (ii) cleave the melt system similarly, (iii) rearrange the boundary conditions to allow interaction of the crystal and melt systems while maintaining the cleaving potentials, and (iv) remove the cleaving potentials from the combined interfacial system.

For steps (i) and (ii), we use the cleaving wall method [17], where the cleaving potential is created by a pair of "cleaving walls," placed on opposite sides of the cleaving plane. The walls are constructed of properly oriented crystal layers that interact with the system particles via a shortrange potential, $\phi(r)$, identical to the system potential, except that the force and potential vanish beyond a cutoff distance $r_{w}$ (see Ref. [17]). Defining $z$ as the coordinate normal to the interface, steps (i) and (ii) are performed by moving the walls towards the cleaving plane from the starting position $z_{i}$, where the cleaving potential is zero, to $z_{f}$ where the system is separated and particles no longer cross the cleaving plane. The work of introducing the cleaving potential is determined by integrating the cleaving force from $z=z_{i}$ to $z_{f}$.

As noted in previous studies [11,17], the structural ordering induced in step (ii) is the principal source of irreversibility. In those studies, it was found that the hysteresis could be reduced to an acceptable level by lengthening the equilibration runs near the transition region; however, for the softer potentials considered here, the hysteresis was found to be significantly larger and more persistent. Modifications of the cleaving potential were found to have little effect. Instead, we have found that the hysteresis can be greatly reduced by cleaving the fluid at densities that are a few percent below the fluid coexistence density $\rho_{f}$. As a result we have modified the cleaving process in step (ii) as follows: The fluid is first stretched in the $z$ direction to reduce the density from $\rho_{f}$ to $\rho<\rho_{f}$. Next, the cleaving potential is introduced. Finally, the system is compressed back to $\rho_{f}$ with the cleaving potential maintained. The additional work of stretching and compressing the system can be calculated by integrating the pressure normal to the interfacial plane with respect to $z$. 
The work required to rearrange the boundary conditions in step (iii) and to remove the cleaving walls in step (iv) is calculated as described in Ref. [17]. The interfacial free energy for the system is calculated by summing the work required in each of the steps (i)-(iv) divided by the area of the created interface.

We have determined $\gamma$ for fcc inverse-power systems with $n=6,7,8,10,12,14,20,30,50$, and 100. In addition, bcc crystal-melt values for $\gamma$ were calculated for $n=6,7$, and 8 . The full results from these simulations are summarized in Table I. The error bounds on the results (shown in parentheses in Table I) are estimated from a combination of statistical error and the magnitude of the residual hysteresis. The precise coexistence conditions (also listed in Table I) were determined in long constant temperature $(N V T)$ simulations, which allowed the interfaces to equilibrate through freezing or melting, while maintaining stress-free conditions in the bulk crystal $[13,17]$. All simulation runs were performed using a Nosé-Hoover thermostat. The system sizes ranged from approximately 15000 particles for $n=6$ to 30000 for $n=$ 100 . For efficiency, the potential was smoothly truncated at a cutoff radius, ranging from $1.2 \sigma$ for $n=100$ to $3.5 \sigma$ for $n=6$ using the truncation discussed in Refs. [13,31].

The scaled interfacial free energies, $\gamma_{1}$, for all systems are plotted in Fig. 1(a) as functions of $1 / n$ (the hard-sphere system is obtained in the limit $1 / n \rightarrow 0$ ). A number of features are striking. First, for $n=6,7$, and 8 where both fcc and bcc interfaces were studied we see that $\gamma$ for the bcc interfaces are approximately $25 \%$ lower than the fcc values for the same potentials. This is consistent both with the recent simulation results for various models for iron [14] and experiments [25] and simulations [26-28] in which preferential nucleation to metastable bcc nuclei is observed in some fcc forming systems. Second, there is a pronounced minimum in $\gamma$ near $n=20(1 / n=0.05)$. A similar minimum in the coexistence densities has been noted [30], which, as Table I shows, occurs at slightly higher values of $n$. Beyond $n=20$, however, the value of $\gamma$ is seen to rise rapidly with increasing $n$.

Figure 1(b) shows the scaled interfacial free energy per interfacial particle, $\bar{\gamma}_{1}$ defined in Eq. (6). The dependence on $n$ for $\bar{\gamma}_{1}$ is much less pronounced than for $\gamma$ itself. This indicates that the primary source of variation in $\gamma$ is the interfacial area per particle (through $\Gamma_{n, c}$ ). Unlike $\gamma, \bar{\gamma}$ is linear for small $1 / n$, which facilitates extrapolation of the data to the hard-sphere limit $(1 / n=0)$. Linear regression for $\bar{\gamma}$ for $1 / n=0.05,0.03 \overline{3}, 0.02$, and 0.01 yields values for the hard-sphere system, which are shown as triangles in Figs. 1(a) and 1(b) and included in the data in Table I. These extrapolated values are about 4\%-6\% lower than the corresponding values reported earlier for the hardsphere system [11,32] but are in closer agreement with results obtained indirectly from experiments on hardsphere-like colloidal systems [33,34]. The current hardsphere results are more consistent than those of Ref. [11] with other recent simulations on fcc systems $[12,17,18]$ in the ordering of the three orientations studied. We regard the current extrapolated hard-sphere values to be the most precise values obtained for this system to date.

While we have determined $\gamma$ only for the [100], [110], and [111] directions, it is possible to extract information as to the full angular dependence of $\gamma$. Defining $\hat{\mathbf{n}}$ as the unit vector perpendicular to the interfacial plane, the orientation dependence of the interfacial free energy, $\gamma(\hat{\mathbf{n}})$, for fcc and bcc crystals can be parametrized by an expansion in cubic harmonics. One such expansion [35], recently applied in a number of studies $[15,17,18]$, is

$$
\begin{aligned}
\gamma(\hat{\mathbf{n}}) / \gamma_{0}= & 1+\epsilon_{1}\left(\sum_{i=1}^{3} n_{i}^{4}-\frac{3}{5}\right) \\
& +\epsilon_{2}\left(\sum_{i=1}^{3} n_{i}^{4}+66 n_{1}^{2} n_{2}^{2} n_{3}^{2}-\frac{17}{7}\right),
\end{aligned}
$$

TABLE I. Summary of results for inverse-power potentials. The values listed for the hard-sphere system $(n=\infty)$ are based on extrapolations of the inverse-power data for $n=100,50,30$, and 20 (see the text). The numbers in parentheses are the estimated errors in the last $\operatorname{digit}(\mathrm{s})$ shown.

\begin{tabular}{rrcccccccccc}
\hline \hline & $n$ & $\Gamma_{n, c}$ & $\Gamma_{n, f}$ & $P_{1}$ & $\gamma_{1}(100)$ & $\gamma_{1}(110)$ & $\gamma_{1}(111)$ & $\gamma_{0}$ & $\epsilon_{1}$ & \multicolumn{1}{c}{$\epsilon_{2}$} & $C_{T}$ \\
\hline fcc & 6 & $2.350(2)$ & $2.323(2)$ & $103.0(2)$ & $0.873(8)$ & $0.796(9)$ & $0.764(11)$ & $0.810(5)$ & $0.20(2)$ & $-0.003(8)$ & $0.60(3)$ \\
& 7 & $1.868(2)$ & $1.838(2)$ & $64.22(16)$ & $0.754(7)$ & $0.695(7)$ & $0.681(7)$ & $0.708(4)$ & $0.16(2)$ & $0.003(7)$ & $0.58(3)$ \\
& 8 & $1.604(2)$ & $1.571(2)$ & $46.39(10)$ & $0.689(8)$ & $0.634(8)$ & $0.622(6)$ & $0.647(4)$ & $0.16(2)$ & $0.004(8)$ & $0.57(2)$ \\
& 10 & $1.334(1)$ & $1.296(1)$ & $30.34(12)$ & $0.620(6)$ & $0.575(7)$ & $0.563(9)$ & $0.584(4)$ & $0.15(2)$ & $0.002(9)$ & $0.54(2)$ \\
& 12 & $1.206(1)$ & $1.163(1)$ & $23.41(4)$ & $0.584(6)$ & $0.545(6)$ & $0.534(7)$ & $0.553(4)$ & $0.14(2)$ & $0.001(8)$ & $0.546(18)$ \\
& 14 & $1.135(1)$ & $1.087(1)$ & $19.82(5)$ & $0.568(6)$ & $0.534(5)$ & $0.519(6)$ & $0.540(3)$ & $0.13(2)$ & $-0.003(7)$ & $0.530(16)$ \\
& 20 & $1.052(1)$ & $0.991(1)$ & $15.15(5)$ & $0.556(5)$ & $0.524(5)$ & $0.511(5)$ & $0.530(3)$ & $0.13(2)$ & $-0.002(6)$ & $0.506(12)$ \\
& 30 & $1.026(1)$ & $0.946(1)$ & $12.94(3)$ & $0.561(5)$ & $0.531(5)$ & $0.516(6)$ & $0.536(3)$ & $0.12(2)$ & $-0.004(7)$ & $0.480(9)$ \\
& 50 & $1.017(1)$ & $0.930(1)$ & $11.95(3)$ & $0.570(4)$ & $0.544(5)$ & $0.530(5)$ & $0.548(3)$ & $0.107(16)$ & $-0.004(6)$ & $0.465(8)$ \\
& 100 & $1.023(1)$ & $0.929(1)$ & $11.56(2)$ & $0.579(5)$ & $0.555(4)$ & $0.543(4)$ & $0.559(3)$ & $0.095(17)$ & $-0.003(5)$ & $0.467(7)$ \\
& $\infty$ & $1.037(1)$ & $0.939(1)$ & $11.57(3)$ & $0.592(7)$ & $0.571(6)$ & $0.557(7)$ & $0.573(5)$ & $0.09(4)$ & $-0.005(8)$ & $0.48(1)$ \\
$\mathrm{bcc}$ & 6 & $2.326(2)$ & $2.299(2)$ & $100.0(2)$ & $0.642(8)$ & $0.607(8)$ & $0.620(7)$ & $0.620(4)$ & $0.06(3)$ & $0.019(7)$ & $0.47(2)$ \\
& 7 & $1.861(2)$ & $1.834(2)$ & $63.88(17)$ & $0.551(7)$ & $0.516(6)$ & $0.532(8)$ & $0.530(4)$ & $0.07(3)$ & $0.021(8)$ & $0.49(2)$ \\
& 8 & $1.607(2)$ & $1.578(2)$ & $47.1(1)$ & $0.497(7)$ & $0.468(7)$ & $0.481(7)$ & $0.480(4)$ & $0.06(3)$ & $0.016(8)$ & $0.48(2)$ \\
\hline \hline
\end{tabular}




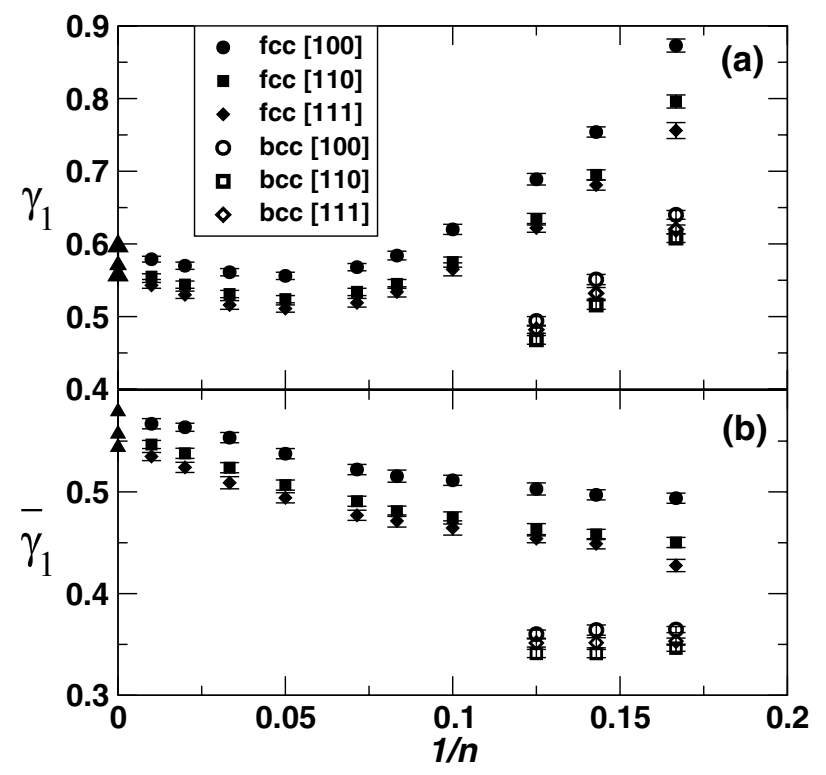

FIG. 1. (a) The scaled crystal-melt interfacial free energies $\gamma_{1}=\gamma^{*} / T^{* 1+2 / n}$ for the inverse-power series as functions of $1 / n$. The open and filled symbols show the data for systems with bcc and fcc crystal structures, respectively. The points at $1 / n=0$ represent extrapolations of the finite $n$ data, as discussed in the text. (b) The same as (a) except for the crystal-melt interfacial free energy per interfacial particle $\bar{\gamma}_{1}=\gamma_{1} \Gamma_{c}^{-2 / 3}$.

where $\left\{n_{1}, n_{2}, n_{3}\right\}$ are the Cartesian components of $\hat{\mathbf{n}}, \gamma_{0}$ is the orientationally averaged interfacial free energy, and $\epsilon_{1}$ and $\epsilon_{2}$ are expansion coefficients that quantify the anisotropy. The results for these parameters are also given in Table I. For the fcc systems, the value of $\epsilon_{1}$ increases with increasing potential range from a value of about 0.1 for hard spheres to 0.2 for $n=6$. The magnitude of $\epsilon_{2}$ for these systems is small and we are unable to resolve the sign of this quantity within the error bars. Consistent with recent studies on iron using the fluctuation method [14], the value of $\epsilon_{1}$ for the bcc systems is smaller than in the corresponding fcc systems, indicating a smaller anisotropy. Within the error bars, the quantity $\epsilon_{1}$ is independent of the range of the potential for the bcc interfaces. The quantity $\epsilon_{2}$ for the bcc systems is seen to be positive with a magnitude of about 0.02 - significantly larger than the fcc value.

Using the values of $\gamma_{0}$ calculated above, we can calculate the Turnbull coefficient from Eq. (1). For fcc systems, $C_{T}$ increases with $1 / n$ (except for a slight minimum at about $1 / n=0.02$ ) and ranges from 0.46 (for $n=50$ ) to 0.6 (for $n=6$ ). This variation is larger than the variation in $\bar{\gamma}_{1}$, indicating that the correlation of $\bar{\gamma}$ with $T_{m}$ defined in Eq. (6) is a better candidate for a general "rule of thumb" for these systems than is Turnbull's rule.

This work was performed using the University of Leicester Mathematical Modelling Centre's supercomputer, which was purchased through the EPSRC strategic equipment initiative. In addition, B. B. L. gratefully acknowledges support from the National Science Foundation under Grant No. CHE9970903.
[1] A. W. Adamson and A.P. Gast, Physical Chemistry of Surfaces (Wiley-Interscience, New York, 1997).

[2] W. A. Tiller, The Science of Crystallization: Microscopic Interfacial Phenomena (Cambridge University Press, New York, 1991).

[3] K. F. Kelton, Solid State Phys. 45, 75 (1991).

[4] L. Granasy and T. Pusztai, J. Chem. Phys. 117, 11121 (2002).

[5] S. Auer and D. Frenkel, Annu. Rev. Phys. Chem. 55, 333 (2004).

[6] A. Karma and W.-J. Rappel, Phys. Rev. E 57, 4323 (1997).

[7] W. J. Boettinger, S. R. Coriell, A. L. Greer, A. Karma, W. Kurz, M. Rappaz, and R. Trivedi, Acta Mater. 48, 43 (2000).

[8] J. M. Howe, Interfaces in Materials (John Wiley \& Sons, New York, 1997).

[9] M. E. Glicksman and N. B. Singh, J. Cryst. Growth 98, 277 (1989).

[10] M. Muschol, D. Liu, and H. Z. Cummins, Phys. Rev. A 46, 1038 (1992).

[11] R. L. Davidchack and B. B. Laird, Phys. Rev. Lett. 85, 4751 (2000).

[12] J. J. Hoyt, M. Asta, and A. Karma, Phys. Rev. Lett. 86, 5530 (2001).

[13] J. R. Morris, Phys. Rev. B 66, 144104 (2002).

[14] D. Y. Sun, M. Asta, and J. J. Hoyt, Phys. Rev. B 69, 174103 (2004).

[15] M. Asta, J.J. Hoyt, and A. Karma, Phys. Rev. B 66, 100101(R) (2002).

[16] J. Q. Broughton and G. H. Gilmer, J. Chem. Phys. 84, 5759 (1986).

[17] R. L. Davidchack and B. B. Laird, J. Chem. Phys. 118, 7651 (2003).

[18] J.R. Morris and X. Song, J. Chem. Phys. 119, 3920 (2003).

[19] D. Turnbull, J. Appl. Phys. 21, 1022 (1950).

[20] A. Sapski, Acta Metall. 4, 583 (1956).

[21] F. Spaepen, Acta Metall. 23, 729 (1975).

[22] F. Spaepen and R. B. Meyer, Scr. Metall. 10, 257 (1976).

[23] B. B. Laird, J. Chem. Phys. 115, 2889 (2001).

[24] W. Ostwald, Z. Phys. Chem. (Leipzig) 22, 289 (1897).

[25] C. Notthoff, B. Feuerbacher, H. Franz, D. M. Herlach, and D. Holland-Moritz, Phys. Rev. Lett. 86, 1038 (2001).

[26] P. R. ten Wolde, M. J. Ruiz-Montero, and D. Frenkel, Phys. Rev. Lett. 75, 2714 (1995).

[27] S. Auer and D. Frenkel, J. Phys. Condens. Matter 14, 7667 (2002).

[28] Y. C. Shen and D. W. Oxtoby, Phys. Rev. Lett. 77, 3585 (1996).

[29] J. P. Hansen and I. R. McDonald, Theory of Simple Liquids (Academic Press, New York, 1976), 1st ed.

[30] R. Agrawal and D. A. Kofke, Phys. Rev. Lett. 74, 122 (1995).

[31] J. Mei and J. W. Davenport, Phys. Rev. B 46, 21 (1992).

[32] S. Auer and D. Frenkel, J. Chem. Phys. 119, 7467 (2003).

[33] D. W. Marr and A. P. Gast, Langmuir 10, 1348 (1994).

[34] T. Palberg, J. Phys. Condens. Matter 11, R323 (1999).

[35] W. R. Fehlner and S.H. Vosko, Can. J. Phys. 54, 2159 (1976). 